\title{
Comparative recalcitrance and extractability of cell wall polysaccharides from cereal (wheat, rye and barley) brans using subcritical water
}

\author{
Supporting Information \\ Andrea C. Ruthes ${ }^{a}, \dagger$, Reskandi C. Rudjito ${ }^{a} \dagger$, Jorge Rencoret ${ }^{b}$, Ana Gutiérrez ${ }^{b}$, José C. \\ del Río ${ }^{b}$ Amparo Jiménez-Quero ${ }^{a}$,Francisco Vilaplana ${ }^{a}$

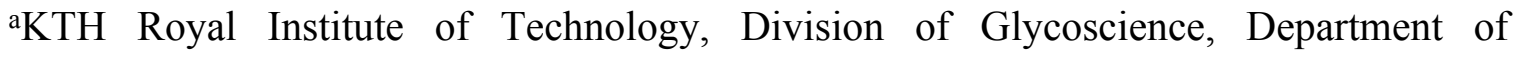 \\ Chemistry, School of Engineering Sciences in Chemistry, Biotechnology, and Health, \\ AlbaNova University Centre, SE-106 91, Stockholm (Sweden)

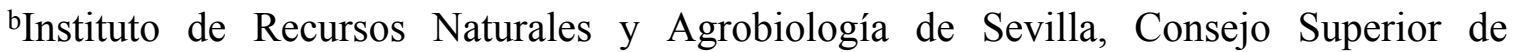 \\ Investigaciones Científicas, Avenida Reina Mercedes, 10, 41012, Seville, Spain

\section{Corresponding Author} \\ *Francisco Vilaplana (franvila@kth.se) \\ $\dagger$ These authors contributed equally to the work
}


Figure S1. Fluorescence microscopy of wheat bran samples. The images were obtained using differential interference contrast (DIC) (left column), DAPI filter (middle column) and DIC merged with DAPI filter (right column).

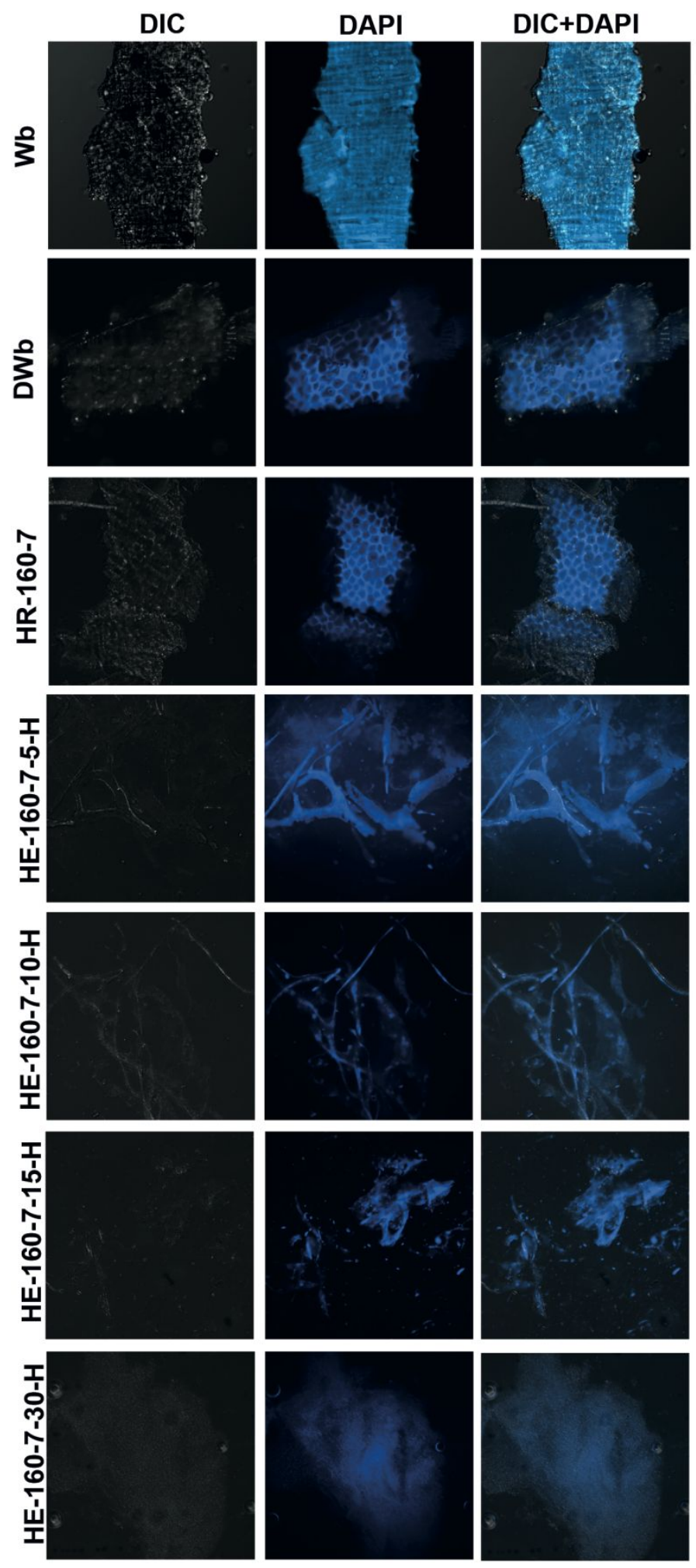


Figure S2. Fluorescence microscopy of rye bran samples. The images were obtained using differential interference contrast (DIC) (left column), DAPI filter (middle column) and DIC merged with DAPI filter (right column).

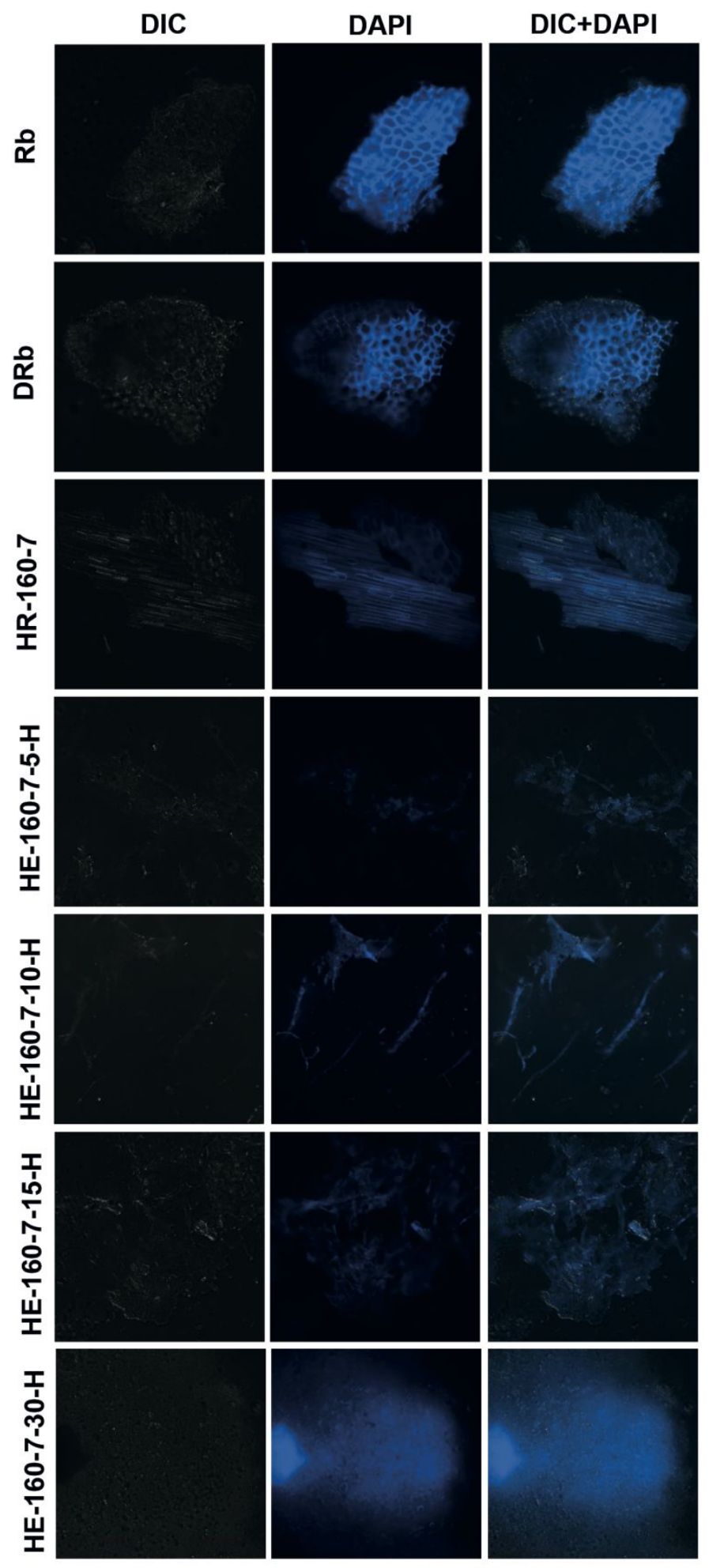


Figure S3. Fluorescence microscopy of barley bran samples. The images were obtained using differential interference contrast (DIC) (left column), DAPI filter (middle column) and DIC merged with DAPI filter (right column).

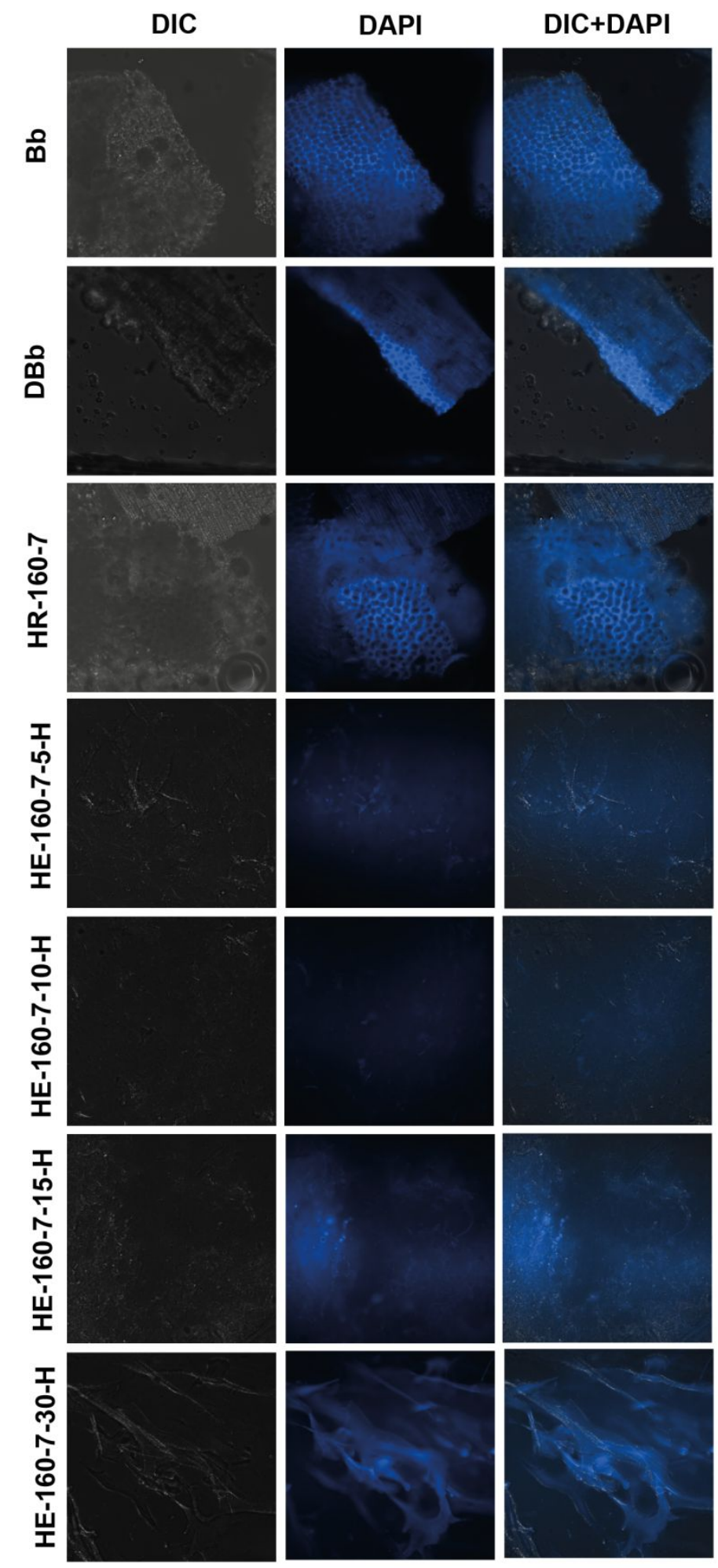


Figure S4. HPLC chromatograms from the phenolic acid profiling of insoluble fractions from wheat bran
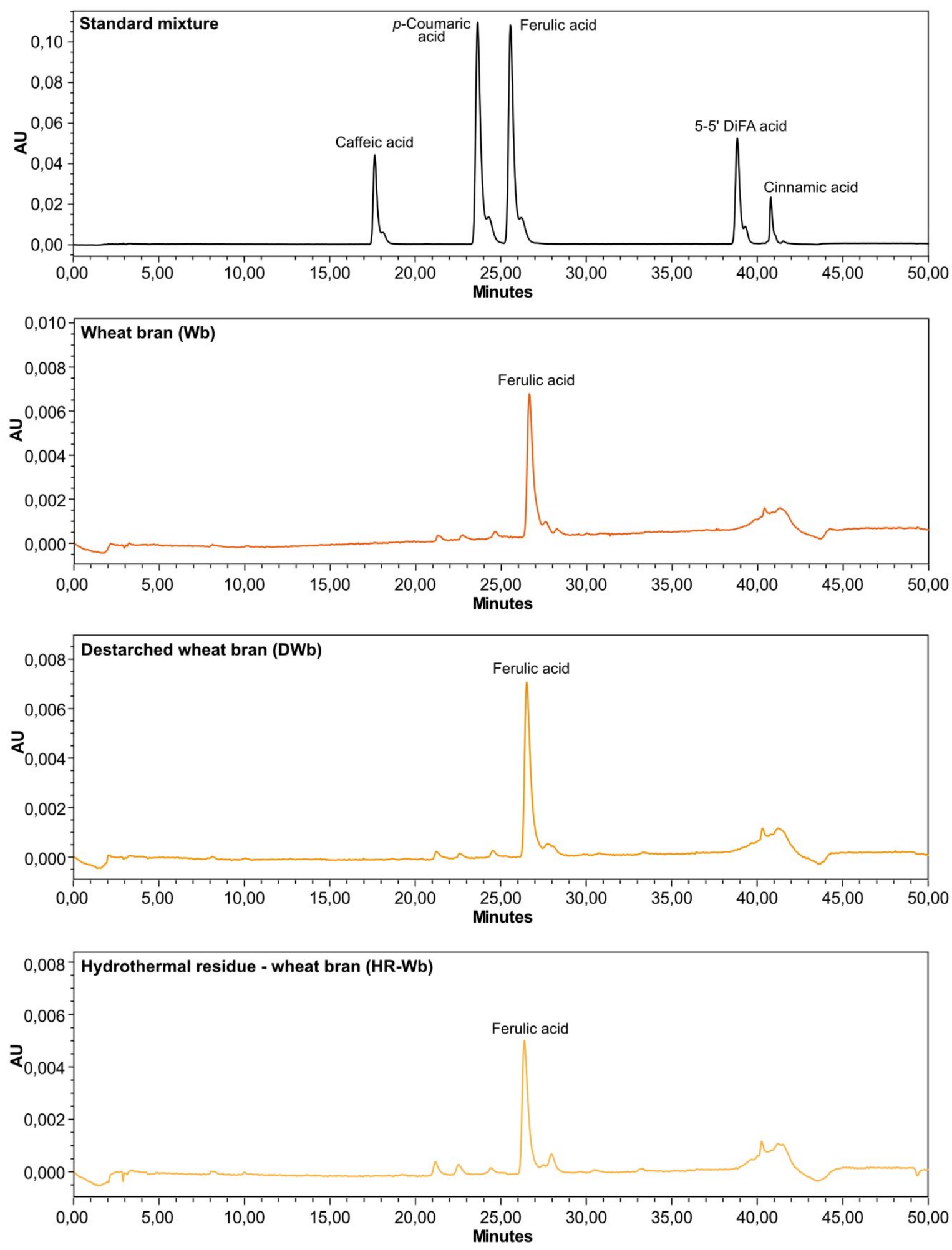
Figure S5. HPLC chromatograms from the phenolic acid profiling of insoluble fractions from rye bran
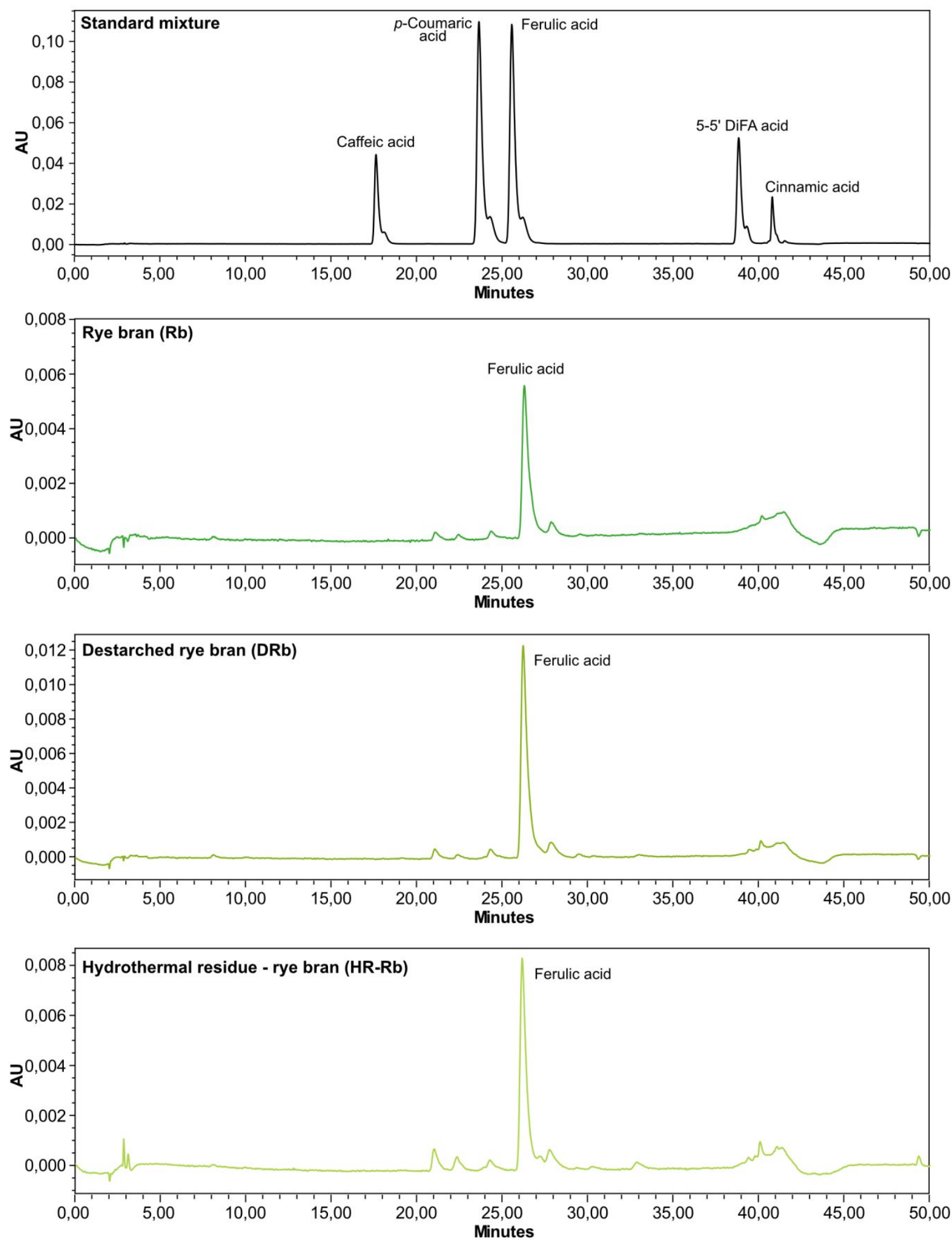
Figure S6. HPLC chromatograms from the phenolic acid profiling of insoluble fractions from barley bran
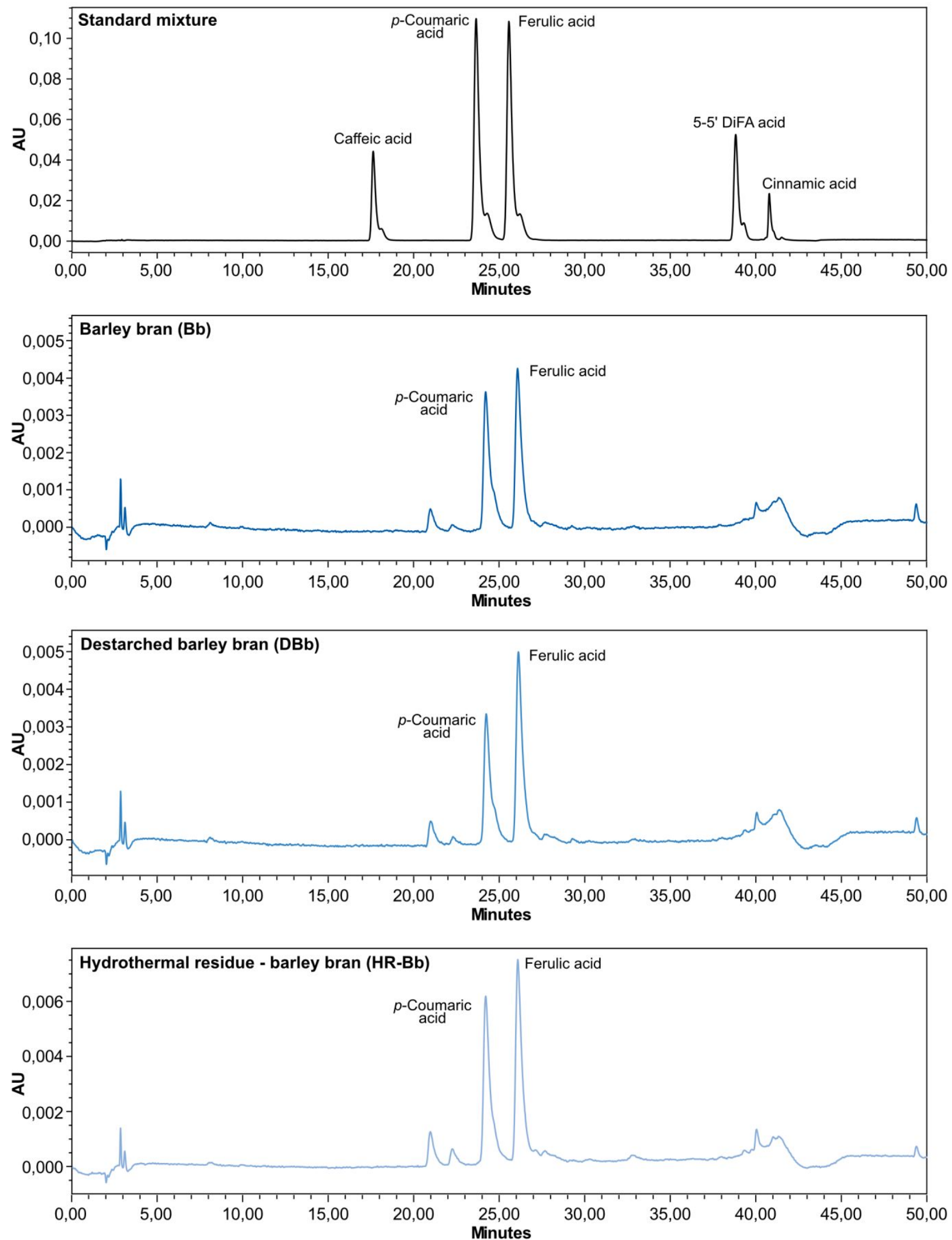
Table S1. Composition of the crude extracts after subcritical water extraction (DW: dryweight basis)

\begin{tabular}{|c|c|c|c|c|c|}
\hline Wheat bran & Wb-5 & Wb-10 & Wb-15 & Wb-30 & Wb-R \\
\hline Yield $(\% \mathrm{DW})^{\mathrm{a}}$ & 12.6 & 7.1 & 3.1 & 4.7 & 49.4 \\
\hline Carbohydrate content $\left(\mathrm{mg} \mathrm{g}^{-1}\right)^{\mathrm{b}}$ & $922.6(2.1)$ & $939.1(20.1)$ & $841.5(14.6)$ & $897.8(6.8)$ & $793.5(6.8)$ \\
\hline Rha (\%) & $0.3(0.0)$ & $0.8(0.1)$ & $1.3(0.2)$ & n.d. & n.d. \\
\hline Ara $(\%)$ & $8.9(0.0)$ & $17.2(0.6)$ & $23.0(0.8)$ & $23.6(0.1)$ & $25.8(0.4)$ \\
\hline Xyl(\%) & $20.6(0.1)$ & $32.0(0.4)$ & $51.2(0.7)$ & $52.0(0.2)$ & $65.2(0.0)$ \\
\hline Man (\%) & $0.3(0.1)$ & $1.0(0.0)$ & $1.0(0.0)$ & $0.3(0.0)$ & $0.7(0.0)$ \\
\hline Gal (\%) & $23.8(0.0)$ & $26.2(0.5)$ & $6.3(1.0)$ & $4.7(0.1)$ & $4.0(0.2)$ \\
\hline Glc (\%) & $46.1(0.1)$ & $22.9(0.5)$ & $17.2(0.6)$ & $19.4(0.3)$ & $4.4(0.1)$ \\
\hline $\mathbf{A X}\left(\mathrm{mg} \mathrm{g}^{-1} \mathrm{DW}\right)^{\mathrm{b}}$ & 271.9 & 461.6 & 624.1 & 679.0 & 721.7 \\
\hline Glucans $\left(\mathrm{mg} \mathrm{g}^{-1}\right)^{\mathrm{b}}$ & 425.5 & 215.2 & 144.7 & 174.2 & 34.5 \\
\hline Other polysaccharides $\left(\mathrm{mg} \mathrm{g}^{-1}\right)^{b}$ & 225.2 & 262.3 & 72.8 & 37.3 & 37.3 \\
\hline Protein content $\left(\mathrm{mg} \mathrm{g}^{-1}\right)^{\mathrm{c}}$ & n.a. & $\mathrm{n}$ & n.a. & n.a. & 120.2 \\
\hline Ferulic acid $\left(\mathrm{mg} \mathrm{g}^{-1}\right)^{\mathrm{d}}$ & $1.5(0.3)$ & $2.9(0.5)$ & $3.8(0.7)$ & $7.3(0.5)$ & $2.9(0.1)$ \\
\hline Rye bran & Rb-5 & Rb-10 & Rb-15 & Rb-30 & $\mathbf{R b}-\mathbf{R}$ \\
\hline Yield $(\% \mathrm{DW})^{\mathrm{a}}$ & 17.4 & 11.2 & 5.8 & 6.1 & 44.7 \\
\hline Carbohydrate content $\left(\mathrm{mg} \mathrm{g}^{-1}\right)^{\mathrm{b}}$ & $943.1(6.5)$ & $951.1(18.1)$ & $973.1(23.5)$ & $922.0(19.9)$ & $819.6(14.2)$ \\
\hline Rha $(9$ & $0.4(0.0)$ & $0.5(0.0)$ & $0.6(0.1)$ & n.d. & n.d. \\
\hline Ara $(\%)$ & $12.5(0.2)$ & $15.2(0.1)$ & $19.6(1.3)$ & $9.8(0.3)$ & $21.5(0.7)$ \\
\hline Xyl(\%) & $30.7(0.9)$ & $28.9(0.8)$ & $55.7(5.7)$ & $68.2(0.7)$ & $60.3(0.3)$ \\
\hline $\operatorname{Man}(\%)$ & $0.7(0.0)$ & $1.7(0.2)$ & $0.5(0.0)$ & n.d. & $0.8(0.2)$ \\
\hline Gal (\%) & $22.3(0.5)$ & $7.6(0.4)$ & $3.5(0.3)$ & $4.9(0.6)$ & $9.4(0.5)$ \\
\hline Glc $(\%)$ & $33.5(0.6)$ & $46.1(0.8)$ & $20.2(4.1)$ & $17.1(1.0)$ & $7.9(0.2)$ \\
\hline $\mathbf{A X}\left(\mathrm{mg} \mathrm{g}^{-1} \mathrm{DW}\right)^{\mathrm{b}}$ & 406.5 & 419.0 & 732.4 & 719.0 & 670.5 \\
\hline Glucans $\left(\mathrm{mg} \mathrm{g}^{-1}\right)^{\mathrm{b}}$ & 316.1 & 438.3 & 196.2 & 157.9 & 65.0 \\
\hline harides $\left(\mathrm{mg} \mathrm{g}^{-1}\right)^{\mathrm{b}}$ & 220.4 & 93.6 & 44.5 & 45.1 & 84.2 \\
\hline Protein con & n.a. & n.a. & n.a & n.a. & 85.1 \\
\hline Ferulic acid $\left(\mathrm{mg} \mathrm{g}^{-1}\right)^{\mathrm{d}}$ & $3.5(0.3)$ & $3.5(0.1)$ & $8.6(0.2)$ & $9.5(0.8)$ & $4.9(0.4)$ \\
\hline Barley & Bb-5 & Bb-10 & Bb-15 & Bb-30 & Bb-R \\
\hline Yield $(\% \mathrm{DW})^{\mathrm{a}}$ & 16.0 & 8.7 & 1.7 & 3 & 51.4 \\
\hline Carbohydrate content $\left(\mathrm{mg} \mathrm{g}^{-1}\right)^{\mathrm{b}}$ & $869.9(8.8)$ & $853.9(13.5)$ & $926.0(15.9)$ & $942.7(15.4)$ & $717.9(5.8)$ \\
\hline Rha $(\%)$ & $0.7(0.0)$ & $1.6(0.2)$ & $1.2(0.1)$ & $0.4(0.0)$ & n.d. \\
\hline Ara $(\%$ & $14.8(4.2)$ & $22.6(2.9)$ & $24.1(0.3)$ & $31.9(0.0)$ & $15.6(0.0)$ \\
\hline $\mathrm{Xyl}(\%$ & $18.0(4.3)$ & $24.6(2.1)$ & $41.3(0.7)$ & $48.7(0.2)$ & $74.9(0.3)$ \\
\hline $\operatorname{Man}($ & $3.1(0.1)$ & $5.9(0.6)$ & $1.9(0.1)$ & $0.3(0.0)$ & $5.3(0.2)$ \\
\hline Gal $(\%$ & $5.4(1.2)$ & $7.4(0.2)$ & $4.5(0.6)$ & $3.9(0.1)$ & $0.7(0.1)$ \\
\hline Glc (\%) & $57.9(9.9)$ & $37.9(4.5)$ & $27.0(0.4)$ & $14.9(0.3)$ & $3.6(0.3)$ \\
\hline $\mathbf{A X}\left(\mathrm{mg} \mathrm{g}^{-1} \mathrm{DW}\right)^{\mathrm{b}}$ & 285.2 & 403.4 & 605.6 & 759.5 & 649.4 \\
\hline Glucans $\left(\mathrm{mg} \mathrm{g}^{-1}\right)^{\mathrm{b}}$ & 504.0 & 323.7 & 250.2 & 140.0 & 25.8 \\
\hline Other po & 80.6 & 126.8 & 70.3 & 43.3 & 42.7 \\
\hline Protein content ( & & & $\mathrm{n} . \mathrm{c}$ & n.a. & 51.5 \\
\hline Ferulic acid $\left(\mathrm{mg} \mathrm{g}^{-1}\right)^{d}$ & $0.8(0.4)$ & $1.9(0.3)$ & $3.1(0.0)$ & $8.3(0.4)$ & $5.2(0.2)$ \\
\hline
\end{tabular}

${ }^{a}$ Extraction yields calculated gravimetrically; ${ }^{\mathrm{b}}$ Carbohydrate content after TFA hydrolysis (for the extracts) or $\mathrm{H}_{2} \mathrm{SO}_{4}$ hydrolysis (for the residues) and HPAEC-PAD; 'Protein content using the Dumas method; ${ }^{\mathrm{d}}$ Ferulic acid content by saponification and GC-MS. 
Table S2. Gravimetric yields after membrane filtration of the crude SWE extracts

\begin{tabular}{|c|c|c|c|c|c|c|c|c|}
\hline \multicolumn{3}{|c|}{ Wheat bran (DWb) } & \multicolumn{3}{|c|}{ Rye bran (DRb) } & \multicolumn{3}{|c|}{ Barley bran (DBb) } \\
\hline Extract & $\mathrm{H}(\%)$ & $\mathrm{L}(\%)$ & Extract & $\mathrm{H}(\%)$ & $\mathrm{L}(\%)$ & Extract & $\mathrm{H}(\%)$ & $\mathrm{L}(\%)$ \\
\hline E-5 & 93.6 & 6.4 & E-5 & 95.3 & 4.7 & E-5 & 98.1 & 1.9 \\
\hline E-10 & 87.4 & 12.6 & $\mathrm{E}-10$ & 92.7 & 7.3 & E-10 & 90.4 & 9.6 \\
\hline E-15 & 92.0 & 8.0 & E-15 & 82.7 & 17.3 & E-15 & 92.4 & 7.6 \\
\hline E-30 & 87.0 & 13.0 & E-30 & 94.8 & 5.2 & E-30 & 92.7 & 7.3 \\
\hline
\end{tabular}

$\mathrm{H}$ : high molar mass fraction; L: low molar mass fraction 
Table S3. Glycosidic linkage analysis of the purified extracts from wheat bran (DWb) after enzymatic purification (destarching) and membrane filtration

\begin{tabular}{|c|c|c|c|c|c|c|c|c|c|}
\hline \multirow{2}{*}{ PMAA } & \multirow{2}{*}{ Proposed linkage } & \multicolumn{2}{|c|}{ DWb-E-5-H } & \multicolumn{2}{|c|}{ DWb-E-10-H } & \multicolumn{2}{|c|}{ DWb-HE-15-H } & \multicolumn{2}{|c|}{ DWb-HE-30-H } \\
\hline & & $\mathrm{AV}$ & STD & $\mathrm{AV}$ & STD & $\mathrm{AV}$ & STD & $\mathrm{AV}$ & STD \\
\hline t-Araf & Araf $f(1 \rightarrow$ & 15.3 & 0.4 & 16.7 & 0.2 & 19.8 & 0.7 & 19.4 & 0.7 \\
\hline 2-Araf & $\rightarrow 2)-\operatorname{Ara} f(1 \rightarrow$ & 0.6 & 0.1 & 0.7 & 0.0 & 1.1 & 0.2 & 1.3 & 0.1 \\
\hline 3-Araf & $\rightarrow 3)$-Araf-(1 $\rightarrow$ & 0.6 & 0.1 & 0.8 & 0.0 & 1.2 & 0.1 & 1.4 & 0.2 \\
\hline 5-Araf & $\rightarrow 5)$-Ara $f-(1 \rightarrow$ & 1.3 & 0.1 & 1.9 & 0.2 & 1.8 & 0.2 & 1.3 & 0.1 \\
\hline 3,5-Araf & $\rightarrow 3,5)$-Araf-(1 $\rightarrow$ & 0.1 & 0.0 & 0.2 & 0.0 & 0.2 & 0.0 & 0.1 & 0.0 \\
\hline 2,5-Ara $f$ & $\rightarrow 2,5)$-Araf-(1 $\rightarrow$ & 0.1 & 0.0 & 0.2 & 0.0 & 0.2 & 0.1 & 0.3 & 0.0 \\
\hline 2,3,5-Ara $f$ & $\rightarrow 2,3,5)$-Araf-(1 $\rightarrow$ & 0.1 & 0.0 & 0.4 & 0.1 & 1.9 & 0.2 & 0.1 & 0.1 \\
\hline Total Ara & & 18.0 & 0.7 & 20.9 & 0.5 & 26.3 & 1.5 & 24.1 & 1.1 \\
\hline $\mathrm{t}-\mathrm{Xyl} p$ & Xyl $p-(1 \rightarrow$ & 1.2 & 0.1 & 1.2 & 0.1 & 1.8 & 0.1 & 1.9 & 0.2 \\
\hline 2-Xylf & $\rightarrow 2)-\mathrm{Xyl} f(1 \rightarrow$ & 0.0 & 0.0 & 0.1 & 0.0 & 0.6 & 0.4 & 0.1 & 0.0 \\
\hline 4-Xylp & $\rightarrow 4)-\mathrm{Xyl} p(1 \rightarrow$ & 27.5 & 0.6 & 30.7 & 1.3 & 39.9 & 2.0 & 39.2 & 3.0 \\
\hline 2,4-Xylp & $\rightarrow 2,4)-\mathrm{Xyl} p(1 \rightarrow$ & 1.7 & 0.1 & 2.3 & 0.5 & 4.1 & 0.2 & 5.0 & 1.0 \\
\hline $3,4-\mathrm{Xyl} p$ & $\rightarrow 3,4)-\mathrm{Xyl} p(1 \rightarrow$ & 3.4 & 0.2 & 5.0 & 0.4 & 7.4 & 0.5 & 8.3 & 0.8 \\
\hline $2,3,4-\mathrm{Xyl} p$ & $\rightarrow 2,3,4)-\mathrm{Xyl} p(1 \rightarrow$ & 3.8 & 0.1 & 4.1 & 0.4 & 5.7 & 0.9 & 5.1 & 0.6 \\
\hline \multicolumn{2}{|c|}{ Total Xyl } & 37.7 & 1.2 & 43.5 & 2.8 & 59.4 & 4.1 & 59.7 & 5.7 \\
\hline $\mathrm{t}-\mathrm{Glc} p$ & Glcp- $(1 \rightarrow$ & 1.7 & 0.1 & 0.5 & 0.1 & 0.6 & 0.0 & 0.2 & 0.0 \\
\hline 3-Glcp & $\rightarrow 3)-\mathrm{Glc} p(1 \rightarrow$ & 3.0 & 0.3 & 2.1 & 0.1 & 2.2 & 0.2 & 2.9 & 0.4 \\
\hline 4-Glc $p$ & $\rightarrow 4)$-Glcp $(1 \rightarrow$ & 20.4 & 0.1 & 14.8 & 0.4 & 5.7 & 0.7 & 9.1 & 0.8 \\
\hline 3,6-Glc $p$ & $\rightarrow 3,6)$-Glc $p(1 \rightarrow$ & 0.1 & 0.0 & 0.0 & 0.0 & 0.6 & 0.8 & 0.0 & 0.0 \\
\hline 4,6-Glc $p$ & $\rightarrow 4,6)$-Glc $p(1 \rightarrow$ & 0.5 & 0.0 & 0.3 & 0.1 & 0.1 & 0.0 & 0.4 & 0.1 \\
\hline \multicolumn{2}{|c|}{ Total Glc } & 25.6 & 0.5 & 17.7 & 0.8 & 9.4 & 1.8 & 12.7 & 1.3 \\
\hline $\mathrm{t}-\mathrm{Gal} p$ & Gal $p-(1 \rightarrow$ & 15.5 & 1.0 & 14.6 & 0.6 & 3.4 & 0.3 & 2.0 & 0.2 \\
\hline 3-Galp & $\rightarrow 3)$-Galp $p(1 \rightarrow$ & 0.4 & 0.0 & 0.9 & 0.1 & 0.3 & 0.0 & 0.2 & 0.0 \\
\hline 2-Galp & $\rightarrow 2)$-Galp $p(1 \rightarrow$ & 0.6 & 0.0 & 0.4 & 0.1 & 0.1 & 0.0 & 0.0 & 0.0 \\
\hline 4-Galp & $\rightarrow 4)$-Galp $p(1 \rightarrow$ & 0.2 & 0.0 & 0.8 & 0.1 & 0.1 & 0.0 & 0.7 & 0.1 \\
\hline 6-Galp & $\rightarrow 6)$-Galp $p(1 \rightarrow$ & 0.2 & 0.0 & 0.1 & 0.1 & 0.1 & 0.0 & 0.1 & 0.0 \\
\hline 2,4-Galp & $\rightarrow 2,4)$-Gal $p(1 \rightarrow$ & 0.5 & 0.0 & 0.1 & 0.1 & 0.0 & 0.0 & 0.0 & 0.0 \\
\hline 3,6-Galp & $\rightarrow 4,6)$-Gal $p(1 \rightarrow$ & 0.5 & 0.0 & 0.2 & 0.1 & 0.2 & 0.1 & 0.1 & 0.0 \\
\hline \multicolumn{2}{|c|}{ Total Gal } & 17.9 & 1.1 & 17.2 & 1.1 & 4.3 & 0.5 & 3.2 & 0.4 \\
\hline t-Man $p$ & 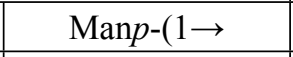 & 0.2 & 0.0 & 0.1 & 0.0 & 0.2 & 0.0 & 0.1 & 0.0 \\
\hline 4-Manp & $\rightarrow 4)-\operatorname{Man} p(1 \rightarrow$ & 0.2 & 0.0 & 0.2 & 0.0 & 0.1 & 0.0 & 0.0 & 0.0 \\
\hline 3,4-Manp & $\rightarrow 3,4)-\operatorname{Man} p(1 \rightarrow$ & 0.1 & 0.1 & 0.2 & 0.1 & 0.1 & 0.0 & 0.1 & 0.0 \\
\hline 2,4-Manp & $\rightarrow 2,4)-\operatorname{Man} p(1 \rightarrow$ & 0.1 & 0.0 & 0.1 & 0.0 & 0.0 & 0.0 & 0.1 & 0.0 \\
\hline \multicolumn{2}{|c|}{ Total Man } & 0.7 & 0.1 & 0.6 & 0.2 & 0.4 & 0.1 & 0.3 & 0.1 \\
\hline 3-Rhap & $\rightarrow 3)-\mathrm{Rha} p(1 \rightarrow$ & 0.1 & 0.0 & 0.1 & 0.0 & 0.2 & 0.0 & 0.1 & 0.0 \\
\hline \multicolumn{2}{|c|}{ Total Rha } & 0.1 & 0.0 & 0.1 & 0.0 & 0.2 & 0.0 & 0.1 & 0.0 \\
\hline \multicolumn{2}{|c|}{$\mathrm{A} / \mathrm{X}$ ratio } & 0.48 & & 0.48 & & 0.44 & & 0.40 & \\
\hline \multicolumn{2}{|r|}{ t:bp } & 1.20 & & 1.06 & & 0.87 & & 0.82 & \\
\hline
\end{tabular}


Table S4. Glycosidic linkage analysis of the purified extracts from rye bran (DRb) after enzymatic purification (destarching) and membrane filtration

\begin{tabular}{|c|c|c|c|c|c|c|c|c|c|}
\hline \multirow{2}{*}{ PMAA } & \multirow{2}{*}{ Proposed linkage } & \multicolumn{2}{|c|}{ DRb-E-5-H } & \multicolumn{2}{|c|}{ DRb-E-10-H } & \multicolumn{2}{|c|}{ DRb-E-15-H } & \multicolumn{2}{|c|}{ DRb-E-30-H } \\
\hline & & $\mathrm{AV}$ & STD & $\mathrm{AV}$ & STD & AV & STD & $\mathrm{AV}$ & STD \\
\hline t-Araf & $\operatorname{Ara} f-(1 \rightarrow$ & 13.7 & 0.2 & 15.2 & 0.6 & 15.6 & 0.6 & 16.0 & 0.7 \\
\hline 2-Araf & $\rightarrow 2)-\operatorname{Araf}(1 \rightarrow$ & 0.5 & 0.0 & 0.5 & 0.0 & 0.6 & 0.0 & 0.7 & 0.1 \\
\hline 3-Araf & $\rightarrow 3)$-Araf-(1 $\rightarrow$ & 0.5 & 0.0 & 0.6 & 0.1 & 0.8 & 0.1 & 0.8 & 0.7 \\
\hline 5-Araf & $\rightarrow 5)$-Araf-(1 $\rightarrow$ & 1.0 & 0.1 & 1.1 & 0.1 & 1.0 & 0.1 & 1.4 & 0.3 \\
\hline 3,5-Araf & $\rightarrow 3,5)$-Araf-( $(1 \rightarrow$ & 0.1 & 0.0 & 0.1 & 0.0 & 0.1 & 0.0 & 0.1 & 0.0 \\
\hline 2,5-Araf & $\rightarrow 2,5)$-Araf-(1 $(1$ & 0.2 & 0.0 & 0.3 & 0.1 & 0.3 & 0.1 & 0.2 & 0.1 \\
\hline 2,3,5-Ara $f$ & $\rightarrow 2,3,5)-\operatorname{Araf}-(1 \rightarrow$ & $<0.1$ & 0.0 & $<0.1$ & 0.0 & 0.1 & 0.0 & 0.1 & 0.0 \\
\hline \multicolumn{2}{|c|}{\begin{tabular}{|c|} 
Total Ara \\
\end{tabular}} & 16.1 & 0.4 & 17.9 & 0.9 & 18.4 & 1.0 & 19.3 & 1.8 \\
\hline $\mathrm{t}-\mathrm{Xyl} f$ & Xylf-(1) & 0.1 & 0.0 & 0.1 & 0.0 & 0.1 & 0.0 & 0.1 & 0.0 \\
\hline $\mathrm{t}-\mathrm{Xyl} p$ & $\mathrm{Xyl} p-(1 \rightarrow$ & 1.3 & 0.1 & 2.3 & 0.0 & 1.1 & 0.1 & 1.3 & 0.1 \\
\hline 2-Xylf & $\rightarrow 2)-\mathrm{Xyl} f(1 \rightarrow$ & 2.6 & 2.2 & 0.1 & 0.0 & 0.1 & 0.1 & 0.1 & 0.0 \\
\hline 4-Xylp & $\rightarrow 4)-\mathrm{Xyl} p(1 \rightarrow$ & 30.5 & 2.4 & 23.0 & 0.8 & 49.0 & 4.9 & 43.1 & 4.7 \\
\hline 2,4-Xylp & $\rightarrow 2,4)-\mathrm{Xyl} p(1 \rightarrow$ & 2.0 & 0.3 & 2.2 & 0.7 & 2.3 & 0.6 & 3.0 & 0.8 \\
\hline 3,4-Xylp & $\rightarrow 3,4)-\mathrm{Xyl} p(1 \rightarrow$ & 6.3 & 0.6 & 6.7 & 0.7 & 11.7 & 0.9 & 11.8 & 1.0 \\
\hline $2,3,4-\mathrm{Xyl} p$ & $\rightarrow 2,3,4)-\mathrm{Xyl} p(1 \rightarrow$ & 2.3 & 0.1 & 3.1 & 0.2 & 2.7 & 0.5 & 2.9 & 0.3 \\
\hline \multicolumn{2}{|c|}{ Total Xyl } & 45.1 & 5.7 & 37.5 & 2.6 & 67.1 & 7.3 & 62.3 & 6.8 \\
\hline t-Glcp & Glc $p-(1 \rightarrow$ & 0.5 & 0.0 & 1.8 & 0.0 & 0.2 & 0.0 & 0.1 & 0.0 \\
\hline 3-Glcp & $\rightarrow 3)$-Glc $p(1 \rightarrow$ & 3.4 & 0.1 & 9.1 & 0.3 & 3.4 & 0.7 & 4.3 & 0.7 \\
\hline 4-Glcp & $\rightarrow 4)$-Glc $p(1 \rightarrow$ & 16.9 & 0.8 & 21.6 & 0.4 & 7.1 & 1.3 & 9.7 & 1.5 \\
\hline 3,6-Glc $p$ & $\rightarrow 3,6)$-Glc $p(1 \rightarrow$ & $<0.1$ & 0.0 & 0.2 & $<0.1$ & 0.0 & $<0.1$ & $<0.1$ & 0.0 \\
\hline 4,6-Glc $p$ & $\rightarrow 4,6)$-Glc $p(1 \rightarrow$ & 0.2 & 0.0 & 0.5 & $<0.1$ & 0.1 & $<0.1$ & 0.1 & 0.0 \\
\hline \multicolumn{2}{|r|}{ Total Glc } & 21.1 & 1.0 & 33.1 & 0.8 & 10.8 & 2.1 & 14.2 & 2.3 \\
\hline t-Galp & Galp- $(1 \rightarrow$ & 15.5 & 0.3 & 8.3 & 0.5 & 2.5 & 0.7 & 2.9 & 0.1 \\
\hline 3-Galp & $\rightarrow 3)$-Galp $p(1 \rightarrow$ & 0.3 & 0.0 & 0.9 & 0.9 & 0.2 & 0.0 & 0.1 & 0.0 \\
\hline 2-Galp & $\rightarrow 2)$-Galp $p(1 \rightarrow$ & 0.4 & 0.0 & 0.5 & 0.0 & 0.1 & 0.0 & $<0.1$ & 0.0 \\
\hline 4-Galp & $\rightarrow 4)$-Galp $p(1 \rightarrow$ & 0.3 & 0.0 & 0.2 & 0.0 & 0.4 & 0.1 & 0.7 & 0.2 \\
\hline 6-Galp & $\rightarrow 6)$-Galp $p(1 \rightarrow$ & 0.3 & 0.0 & 0.1 & 0.0 & 0.1 & 0.0 & 0.1 & 0.0 \\
\hline 2,4-Gal $p$ & $\rightarrow 2,4)-\mathrm{Gal} p(1 \rightarrow$ & 0.1 & 0.0 & 0.3 & 0.0 & $<0.1$ & 0.0 & $<0.1$ & 0.0 \\
\hline 3,6-Gal $p$ & $\rightarrow 4,6)-\mathrm{Gal} p(1 \rightarrow$ & 0.1 & 0.0 & 0.4 & 0.1 & 0.2 & 0.0 & 0.2 & 0.0 \\
\hline \multicolumn{2}{|r|}{ Total Gal } & 17.0 & 0.4 & 10.7 & 1.6 & 3.4 & 1.0 & 4.0 & 0.3 \\
\hline t-Man $p$ & $\operatorname{Man} p-(1 \rightarrow$ & 0.2 & 0.2 & 0.1 & 0.0 & $<0.1$ & 0.0 & $<0.1$ & 0.0 \\
\hline 4-Manp & $\rightarrow 4)-\operatorname{Man} p(1 \rightarrow$ & 0.2 & 0.0 & 0.4 & 0.0 & 0.1 & 0.0 & 0.1 & 0.0 \\
\hline 3,4-Man $p$ & $\rightarrow 3,4)-\operatorname{Man} p(1 \rightarrow$ & 0.2 & 0.1 & 0.2 & 0.1 & 0.1 & 0.0 & 0.1 & 0.0 \\
\hline 2,4-Man $p$ & $\rightarrow 2,4)-\operatorname{Man} p(1 \rightarrow$ & 0.1 & 0.0 & 0.1 & 0.0 & $<0.1$ & 0.0 & $<0.1$ & 0.0 \\
\hline \multicolumn{2}{|c|}{ Total Man } & 0.6 & 0.4 & 0.7 & 0.1 & 0.2 & 0.1 & 0.2 & 0.1 \\
\hline 2-Rhap & $\rightarrow 2)-R h a p(1 \rightarrow$ & $<0.1$ & 0.0 & $<0.1$ & 0.0 & $<0.1$ & 0.0 & $<0.1$ & 0.0 \\
\hline 3-Rhap & $\rightarrow 3)$-Rhap $(1 \rightarrow$ & $<0.1$ & 0.0 & $<0.1$ & 0.0 & 0.1 & 0.0 & $<0.1$ & 0.0 \\
\hline \multicolumn{2}{|c|}{ Total Rha } & 0.1 & 0.0 & $<0.1$ & 0.0 & 0.1 & 0.0 & $<0.1$ & 0.0 \\
\hline \multicolumn{2}{|c|}{$\mathrm{A} / \mathrm{X}$ ratio } & 0.36 & & 0.48 & & 0.27 & & 0.31 & \\
\hline \multicolumn{2}{|r|}{ t:bp } & 1.06 & & 1.01 & & 0.80 & & 0.78 & \\
\hline
\end{tabular}


Table S5. Glycosidic linkage analysis of the purified extracts from barley bran (DBb) after enzymatic purification (destarching) and membrane filtration

\begin{tabular}{|c|c|c|c|c|c|c|c|c|c|}
\hline \multirow{2}{*}{ PMAA } & \multirow{2}{*}{ Proposed linkage } & \multicolumn{2}{|c|}{ DBb-E-5-H } & \multicolumn{2}{|c|}{ DBb-E-10-H } & \multicolumn{2}{|c|}{ DBb-E-15-H } & \multicolumn{2}{|c|}{ DBb-E-30-H } \\
\hline & & $\mathrm{AV}$ & STD & $\mathrm{AV}$ & STD & $\mathrm{AV}$ & STD & $\mathrm{AV}$ & STD \\
\hline t-Araf & $\operatorname{Ara} f-(1 \rightarrow$ & 9.3 & 0.8 & 14.0 & 0.7 & 26.0 & 0.0 & 22.3 & 0.5 \\
\hline 2-Araf & $\rightarrow 2)-\operatorname{Ara} f(1 \rightarrow$ & 0.3 & 0.0 & 0.6 & 0.0 & 1.1 & 0.0 & 1.4 & 0.1 \\
\hline 3-Araf & $\rightarrow 3)$-Araf- $(1 \rightarrow$ & 0.5 & 0.1 & 0.9 & 0.0 & 1.4 & 0.0 & 1.6 & 0.3 \\
\hline 5-Araf & $\rightarrow 5)$-Araf-(1 $\rightarrow$ & 0.7 & 0.0 & 1.7 & 0.1 & 1.8 & 0.0 & 1.8 & 0.3 \\
\hline 3,5-Araf & $\rightarrow 3,5)-\operatorname{Ara} f-(1 \rightarrow$ & 0.1 & 0.0 & 0.2 & 0.0 & 0.1 & 0.0 & 0.1 & 0.0 \\
\hline 2,5-Araf & $\rightarrow 2,5)$-Araf-(1 $\rightarrow$ & 0.3 & 0.1 & 0.3 & 0.0 & 0.2 & 0.0 & 0.3 & 0.2 \\
\hline 2,3,5-Araf & $\rightarrow 2,3,5)-\operatorname{Ara} f-(1 \rightarrow$ & 0.1 & 0.0 & 0.1 & 0.0 & 0.2 & 0.0 & 0.1 & 0.1 \\
\hline \multicolumn{2}{|c|}{ Total Ara } & 11.3 & 1.0 & 17.6 & 0.9 & 31.0 & 0.0 & 27.4 & 1.4 \\
\hline $\mathrm{t}-\mathrm{Xyl} f$ & Xylf-(1) & 0.1 & 0.0 & 0.1 & 0.0 & 0.2 & 0.0 & 0.3 & 0.1 \\
\hline $\mathrm{t}-\mathrm{Xyl} p$ & $\mathrm{Xyl} p-(1 \rightarrow$ & 0.7 & 0.1 & 1.4 & 0.1 & 2.0 & 0.0 & 2.3 & 0.2 \\
\hline 2-Xyl $f$ & $\rightarrow 2)-\mathrm{Xyl} f(1 \rightarrow$ & $<0.1$ & 0.0 & $<0.1$ & 0.0 & 0.1 & 0.0 & 0.1 & 0.1 \\
\hline 4-Xylp & $\rightarrow 4)-\mathrm{Xyl} p(1 \rightarrow$ & 8.7 & 1.3 & 7.8 & 0.6 & 28.3 & 0.0 & 36.0 & 2.6 \\
\hline $2,4-\mathrm{Xyl} p$ & $\rightarrow 2,4)-\mathrm{Xyl} p(1 \rightarrow$ & 1.2 & 0.2 & 1.6 & 0.1 & 2.9 & 0.0 & 4.9 & 0.3 \\
\hline 3,4-Xylp & $\rightarrow 3,4)-\mathrm{Xyl} p(1 \rightarrow$ & 2.4 & 0.7 & 3.8 & 0.2 & 7.9 & 0.0 & 9.6 & 0.5 \\
\hline 2,3,4-Xyl $p$ & $\rightarrow 2,3,4)-\mathrm{Xyl} p(1 \rightarrow$ & 2.3 & 0.7 & 3.0 & 0.3 & 4.2 & 0.0 & 5.3 & 0.3 \\
\hline \multicolumn{2}{|c|}{ Total Xyl } & 15.4 & 3.1 & 17.7 & 1.4 & 45.6 & 0.0 & 58.4 & 4.1 \\
\hline t-Glcp & Glcp- $(1 \rightarrow$ & 1.9 & 0.1 & 1.3 & 0.1 & 0.3 & 0.0 & 0.2 & 0.0 \\
\hline 3-Glcp & $\rightarrow 3)-\operatorname{Glc} p(1 \rightarrow$ & 11.7 & 1.6 & 15.5 & 0.4 & 4.8 & 0.0 & 2.4 & 0.5 \\
\hline 4-Glcp & $\rightarrow 4)$-Glc $p(1 \rightarrow$ & 43.3 & 3.5 & 33.9 & 1.3 & 11.5 & 0.0 & 7.3 & 1.2 \\
\hline $4,6-\mathrm{Glc} p$ & $\rightarrow 4,6)-$ Glc $p(1 \rightarrow$ & 0.7 & 0.1 & 0.4 & 0.1 & 0.2 & 0.0 & 0.2 & 0.1 \\
\hline \multicolumn{2}{|c|}{ Total GIc } & 57.9 & 5.3 & 51.4 & 1.9 & 17.0 & 0.0 & 10.2 & 1.9 \\
\hline $\mathrm{t}-\mathrm{Gal} p$ & Galp-(1) & 11.1 & 1.1 & 8.6 & 0.7 & 3.3 & 0.0 & 2.0 & 0.2 \\
\hline 3-Galp & $\rightarrow 3)$-Gal $p(1 \rightarrow$ & 0.4 & 0.1 & 0.4 & 0.0 & 0.4 & 0.0 & 0.3 & 0.1 \\
\hline 2-Galp & $\rightarrow 2)$-Gal $p(1 \rightarrow$ & 0.3 & 0.0 & 0.3 & 0.0 & 0.1 & 0.0 & 0.0 & 0.0 \\
\hline 4-Gal $p$ & $\rightarrow 4)$-Gal $p(1 \rightarrow$ & 0.3 & 0.0 & 0.6 & 0.0 & 0.5 & 0.0 & 0.7 & 0.2 \\
\hline 6-Galp & $\rightarrow 6)-\mathrm{Gal} p(1 \rightarrow$ & 0.1 & 0.0 & 0.1 & 0.0 & 0.1 & 0.0 & 0.1 & 0.0 \\
\hline 2,4-Gal $p$ & $\rightarrow 2,4)$-Galp $(1 \rightarrow$ & 0.3 & 0.0 & 0.1 & 0.1 & 0.1 & 0.0 & 0.0 & 0.0 \\
\hline 3,6-Galp & $\rightarrow 4,6)-\mathrm{Gal} p(1 \rightarrow$ & 0.6 & 0.0 & 0.4 & 0.0 & 0.5 & 0.0 & 0.5 & 0.1 \\
\hline \multicolumn{2}{|c|}{ Total Gal } & 13.0 & 1.4 & 10.6 & 0.9 & 4.9 & 0.0 & 3.6 & 0.6 \\
\hline t-Man $p$ & $\operatorname{Man} p-(1 \rightarrow$ & $<0.1$ & 0.0 & $<0.1$ & 0.0 & $<0.1$ & 0.0 & $<0.1$ & 0.0 \\
\hline 4-Manp & $\rightarrow 4)-\operatorname{Man} p(1 \rightarrow$ & 1.6 & 0.1 & 2.1 & 0.2 & 0.7 & 0.0 & 0.2 & 0.0 \\
\hline 3,4-Manp & $\rightarrow 3,4)-\operatorname{Man} p(1 \rightarrow$ & 0.5 & 0.1 & 0.2 & 0.1 & 0.5 & 0.0 & 0.1 & 0.0 \\
\hline 2,4-Manp & $\rightarrow 2,4)-\operatorname{Man} p(1 \rightarrow$ & 0.2 & 0.1 & 0.1 & 0.0 & 0.1 & 0.0 & $<0.1$ & 0.0 \\
\hline 4,6-Man $p$ & $\rightarrow 4,6)-\operatorname{Man} p(1 \rightarrow$ & $<0.1$ & 0.0 & 0.1 & 0.1 & $<0.1$ & 0.0 & $<0.1$ & 0.0 \\
\hline \multicolumn{2}{|c|}{ Total Man } & 2.4 & 0.3 & 2.6 & 0.4 & 1.3 & 0.0 & 0.3 & 0.1 \\
\hline 2-Rhap & $\rightarrow 2)$-Rhap $(1 \rightarrow$ & $<0.1$ & 0.0 & $<0.1$ & 0.0 & 0.1 & 0.0 & $<0.1$ & 0.0 \\
\hline 3-Rhap & $\rightarrow 3)$-Rhap $(1 \rightarrow$ & 0.1 & 0.0 & 0.1 & 0.0 & 0.1 & 0.0 & 0.0 & 0.0 \\
\hline \multicolumn{2}{|c|}{ Total Rha } & 0.1 & 0.0 & 0.1 & 0.0 & 0.2 & 0.0 & 0.1 & 0.0 \\
\hline \multicolumn{2}{|c|}{$\mathrm{A} / \mathrm{X}$ ratio } & 0.73 & & 1.00 & & 0.68 & & 0.47 & \\
\hline \multicolumn{2}{|r|}{ t:bp } & 1.15 & & 1.23 & & 1.35 & & 0.89 & \\
\hline
\end{tabular}


Table S6. Phenolic acid composition of the insoluble bran fractions

\begin{tabular}{|l|c|c|c|}
\hline & Initial bran & Destarched bran & Residue \\
\hline Wheat bran & Wb & DWb & HR-Wb \\
\hline Total phenolic acid content $\left(\mathrm{mg} \mathrm{g}^{-1}\right)^{\mathrm{a}}$ & $2.9(0.5)$ & $3.9(0.5)$ & $2.7(0.2)$ \\
\hline Caffeic (\%) & 0.0 & 0.0 & 0.0 \\
$\boldsymbol{p}$-Coumaric (\%) & $3.1(0.2)$ & $2.5(0.6)$ & $3.6(0.1)$ \\
Ferulic (\%) & $96.9(0.2)$ & $97.5(0.6)$ & $96.4(0.1)$ \\
Sinapic (\%) & 0.0 & 0.0 & 0.0 \\
5-5' DiFA (\%) & 0.0 & 0.0 & 0.0 \\
Cinnamic (\%) & 0.0 & 0.0 & 0.0 \\
\hline Rye bran & Rb & DRb & HR-Rb \\
\hline Total phenolic acid content $\left(\mathrm{mg} \mathrm{g}^{-1}\right)^{\mathrm{a}}$ & $3.1(0.2)$ & $6.3(0.3)$ & $5.2(0.3)$ \\
\hline Caffeic (\%) & 0.0 & 0.0 & 0.0 \\
p-Coumaric (\%) & $2.8(0.4)$ & $3.3(0.1)$ & $5.2(1.1)$ \\
Ferulic (\%) & $97.2(0.4)$ & $95.5(0.3)$ & $92.7(0.6)$ \\
Sinapic (\%) & 0.0 & 0.0 & 0.0 \\
5-5' DiFA (\%) & 0.0 & $1.2(0.2)$ & $2.1(0.5)$ \\
Cinnamic (\%) & 0.0 & 0.0 & 0.0 \\
\hline Barley bran & Bb & DBb & HR-Bb \\
\hline Total phenolic acid content $\left(\mathrm{mg} \mathrm{g}^{-1}\right)^{\mathrm{a}}$ & $3.6(0.3)$ & $4.4(0.8)$ & $7.6(0.2)$ \\
\hline Caffeic (\%) & 0.0 & 0.0 & 0.0 \\
p-Coumaric (\%) & $47.4(2.2)$ & $42.2(0.9)$ & $46.6(0.6)$ \\
Ferulic (\%) & $52.6(2.2)$ & $57.8(0.9)$ & $52.4(0.4)$ \\
Sinapic (\%) & 0.0 & 0.0 & 0.0 \\
5-5' DiFA (\%) & 0.0 & 0.0 & $1.0(0.2)$ \\
Cinnamic (\%) & 0.0 & 0.0 & 0.0 \\
\hline
\end{tabular}

${ }^{a}$ Total phenolic acid content was determined by saponification followed by HPLC analysis; ${ }^{b}$ DiFA refers to ferulic acid dehydrodimer 